\title{
Estudo do tratamento do edema macular difuso do diabético com triancinolona intravítrea e fotocoagulação
}

\author{
Use of intravitreal triamcinolone and laser photocoagulation for the treatment \\ of diffuse diabetic macular edema
}

\author{
Fábio Petersen Saraiva ${ }^{1}$ \\ Márcia Silva Queiroz ${ }^{2}$ \\ Patricia Grativol Costa ${ }^{3}$ \\ Fábio Gasparin ${ }^{4}$ \\ Yoshitaka Nakashima $^{5}$
}

\begin{tabular}{|l|}
\hline RESUMO \\
\hline Objetivo: Avaliar a eficácia do uso intravítreo da triancinolona acetonida \\
associada a fotocoagulação com laser no tratamento do edema macular \\
difuso diabético. Comparar esta associação terapêutica com o uso isolado \\
de cada tratamento. Métodos: Após controle clínico sistêmico, trinta \\
pacientes portadores de edema macular difuso diabético foram divididos \\
em 3 grupos de tratamento: (1) fotocoagulação macular em grade com laser \\
de argônio; (2) aplicação intravítrea de 4 mg de triancinolona acetonida; \\
(3) associação dos itens anteriores. O seguimento foi realizado em inter- \\
valos predeterminados de um dia, uma semana e mensalmente, até comple- \\
tar 6 meses. Foram analisados os parâmetros: acuidade visual corrigida \\
LogMAR, espessura macular central, volume macular total e pressão intra- \\
ocular. Resultados: A fotocoagulação com laser não reduziu de forma \\
estatisticamente significativa a espessura macular central e o volume \\
macular total. Esta redução foi significativa e estatisticamente semelhante \\
nos outros dois grupos. Todos os grupos apresentaram melhora da acui- \\
dade visual, entretanto, o grupo que recebeu a associação do laser com a \\
triancinolona intravítrea obteve maior porcentagem de pacientes com \\
ganho de 10 ou mais letras de visão. Conclusão: O uso simultâneo da \\
fotocoagulação com a triancinolona intravítrea pode ser considerado uma \\
opção terapêutica para o edema macular difuso diabético.
\end{tabular}

Descritores: Retinopatia diabética/complicações; Acuidade visual; Complicações do diabetes; Edema macular/quimioterapia; Triancinolona acetonida/uso terapêutico; Triancinolona acetonida/administração \& dosagem; Coagulação por laser

\section{INTRODUÇ̃̃O}

A retinopatia diabética proliferativa e o edema macular diabético estão entre as principais causas de baixa visual e cegueira legal do mundo ${ }^{(1)}$. A terapêutica do edema macular clinicamente significativo (EMCS) do diabetes consiste, classicamente, na fotocoagulação com laser dos microaneurismas $^{(2)}$. Entretanto, na forma difusa do EMCS não é possível a localização exata dos pontos de extravasamento, pois, todo o leito microvascular está alterado, tornando a fotocoagulação mais complicada e muitas vezes ineficaz. É comum, nestes casos, a repetição do laser ${ }^{(3-4)}$. Este tratamento com laser é feito na forma de uma grade ou rede ("grid"). A necessidade de sucessivas sessões de laser aumenta o risco de complicações relacionadas a este tratamento ${ }^{(5-6)}$.

A triancinolona acetonida (TA) é um corticosteróide de depósito usado na oftalmologia há mais de $40 \operatorname{anos}^{(7)}$ e, a sua administração intravítrea 
(IVTA), vem sendo amplamente estudada para tratamento de diversas afecções oculares, inclusive como alternativa ao laser em algumas situações ${ }^{(4,8-9)}$.

A eficácia da IVTA no tratamento do EMCS difuso do diabético já foi comprovada e, alguns trabalhos demonstraram, inclusive, resultados visuais significativamente melhores do que o grid macular ${ }^{(4,10)}$. Alguns autores demonstraram resultados positivos da associação do grid macular com a triancinolona subtenoniana no tratamento do edema macular diabéti$\mathrm{co}^{(11-12)}$. Outros trabalhos estudaram o efeito do grid macular após 3-12 semanas da IVTA ${ }^{(10,13)}$. Todavia, não encontramos na literatura estudos sobre a aplicação simultânea do grid macular com a IVTA no tratamento do EMCS difuso diabético.

O objetivo deste trabalho foi avaliar a eficácia da administração simultânea da IVTA, com a fotocoagulação macular, no tratamento do edema macular difuso diabético e comparar esta associação terapêutica com o uso isolado de cada tratamento.

\section{MÉTODOS}

Os pacientes portadores de edema macular difuso diabético foram triados, por médicos estagiários e residentes, entre os usuários do ambulatório de Retina e Vítreo do Hospital das Clínicas da Universidade de São Paulo. Todos os pacientes triados foram encaminhados para avaliação endocrinológica e somente foram liberados para iniciarem o protocolo após exame clínico adequado, considerado por glicemia de jejum inferior a $150 \mathrm{mg} / \mathrm{dL}$, hemoglobina glicada menor que $7,5 \%$, pressão arterial inferior a $140 \times 90 \mathrm{mmHg}$ e creatinina $\leq 2 \mathrm{mg} / \mathrm{dL}$. Aqueles identificados como virgens de tratamento foram submetidos a uma sessão de grid macular antes do encaminhamento ao endocrinologista.

A avaliação oftalmológica incluiu: exame refracional com medida da acuidade visual corrigida; biomicroscopia; tonometria (PIO) e mapeamento da retina com lente de 78 e 20 dioptrias. A refração e a medida da acuidade visual foram realizadas com caixa e armação de provas, numa distância de dois metros, utilizando caixa retroiluminada e as tabelas preconizadas no ETDRS ${ }^{(2)}$. O modelo de medida da acuidade visual utilizado foi o logMAR ("logarithm of minimal angle of resolution”). A avaliação do edema macular foi realizada por meio de biomicroscopia de fundo, angiografia digital e medida da espessura macular central e do volume macular total com aparelho de tomografia de coerência óptica (OCT; Stratus OCT $^{\text {TM }}$ Model 3000, Version 4.0.1, Carl Zeiss Meditec Inc. Dublin, CA, USA). Os critérios para caracterização do edema macular difuso foram a presença de um espessamento generalizado da mácula observada pela biomicroscopia de fundo e a evidência, no angiograma, de extravasamento macular difuso do contraste. O protocolo de exame utilizado para varredura da retina com o OCT foi o "Fast Macular"; registrou-se o valor médio de três medidas.

Para a inclusão no estudo, os pacientes deviam apresentar um edema macular refratário a pelo menos uma sessão de grid macular há no mínimo três meses. Os pacientes, também, não deveriam apresentar evidências de tração vítreo-retiniana ou membrana epirretiniana. Aqueles virgens de tratamento que obtiveram melhora com o grid e aqueles em que o controle clínico e/ou laboratorial das condições sistêmicas não foi possível, foram excluídos do estudo. Da mesma forma, foram excluídos os portadores de outras afecções oculares que pudessem acarretar déficit visual.

Foi estudado um olho de cada paciente. Caso ambos os olhos cumprissem os critérios do estudo, apenas um era sorteado para participação no trabalho. O olho contralateral não foi utilizado no estudo porque nem todos os pacientes apresentavam edema macular difuso em ambos os olhos. Os pacientes selecionados foram distribuídos de forma randomizada em três diferentes grupos de tratamento:

Grupo Laser - fotocoagulação macular em grade com laser de argônio;

Grupo TA - administração intravítrea de $4 \mathrm{mg}$ de triancinolona acetonida;

Grupo Laser+TA - associação do laser com a triancinolona intravítrea.

A fotocoagulação macular foi aplicada com uma mira de $100 \mu \mathrm{m}$, intervalo de 0,1 segundo e potência necessária para uma discreta marca branco-acinzentada na retina espessada, sendo respeitado uma distância de uma a duas marcas entre elas.

Os pacientes do grupo Laser+TA recebeu, primeiramente, a fotocoagulação e, alguns minutos depois, a IVTA.

Para o estudo foi utilizada a dose de $4 \mathrm{mg}$ de TA que representa $0,1 \mathrm{ml}$ do frasco de concentração $40 \mathrm{mg} / \mathrm{ml}$ (Kena$\log -40^{\circledR}$; Bristol-Myers).

Os procedimentos para a aplicação da triancinolona seguiram parâmetros de consenso já publicados ${ }^{(14)}$.

O acompanhamento dos pacientes ocorreu em intervalos pré-determinados de um dia, uma semana, e mensalmente até completar seis meses; Foram analisados os seguintes parâmetros: espessura macular central, volume macular total, acuidade visual corrigida $\log$ MAR e pressão intra-ocular. As complicações foram registradas e devidamente tratadas.

Os dados quantitativos referentes à acuidade visual também foram transformados em dados qualitativos e distribuídos numa escala ordinal de ganhos ou perdas de letras de visão, cujas categorias foram: perda de 10 ou mais letras; perda de 5 a 9 letras; estável; ganho de 5 a 9 letras; ganho de 10 ou mais letras. Isto foi feito para possibilitar uma melhor comparação com os dados da literatura. Baseando-se nesta mesma finalidade, verificamos o número de pacientes que obtiveram PIO maior que $21 \mathrm{mmHg}$ e o número de pacientes que tiveram a PIO elevada em $5 \mathrm{mmHg}$ ou mais após tratamento.

Todos os pacientes concordaram em participar do estudo e assinaram o termo de consentimento livre e esclarecido aprovado pela comissão de ética do Hospital.

\section{Métodos Estatísticos}

A homogeneidade entre os grupos foi testada para as variáveis: idade, sexo, presença de pseudofacia, realização de 
panfotocoagulação prévia e número de vezes que foi realizado grid macular. Para a variável quantitativa idade, utilizou-se a Análise de Variância a um fator. As demais variáveis qualitativas foram analisadas pelo Teste Exato de Fisher (bicaudal).

Para averiguar o comportamento dos grupos considerando as variáveis edema macular central, volume macular total, acuidade visual e pressão intra-ocular fez-se uso da técnica Análise de Variância com medidas repetidas.

Após distribuição em escala ordinal de ganhos ou perdas de letras de visão, a evolução da acuidade visual também foi testada pelo Teste Exato de Fisher (bicaudal). Este mesmo teste foi utilizado na análise, entre os grupos, da quantidade de pacientes com PIO maior que $21 \mathrm{mmHg}$ ou com elevação pressórica maior ou igual a $5 \mathrm{mmHg}$ após tratamento.

O nível de significância utilizado para os testes foi de $5 \%$.

\section{RESULTADOS}

Quarenta e oito pacientes foram triados para o estudo. Desta amostra, foram excluídos sete pela impossibilidade de controle clínico e/ou laboratorial do diabetes mellitus; quatro por terem melhorado o edema após três meses da fotocoagulação primária; dois por serem analfabetos e incapacitados para o exame de acuidade visual; dois devido a insuficiência renal crônica; um por ter oclusão venosa retiniana; um pelo diagnóstico de glaucoma e um por ter escavação suspeita de glaucoma. Foram estudados, portanto, 30 pacientes $(10 \mathrm{em}$ cada grupo), sendo 14 mulheres e 16 homens. A idade média foi $64 \pm 8,8$ anos. O intervalo médio entre o controle clínico do paciente e o início do estudo foi de 2,4 $\pm 1,9$ meses. Dos olhos estudados, 7 eram pseudofácicos, 7 haviam sido submetidos a panfotocoagulação prévia e 23 foram tratados previamente com apenas uma sessão de grid macular. Os grupos não diferiram em relação a estas variáveis citadas. Por meio do OCT foi possível observar a presença de edema macular com formação de cistos em 19 pacientes e um pequeno descolamento seroso subfoveal associado ao EMD em apenas um caso.

Um paciente do grupo Laser+TA faleceu entre o $4^{\circ}$ e $5^{\circ}$ mês de seguimento devido a um infarto agudo do miocárdio e um paciente do grupo TA apresentou uma uveíte anterior aguda entre o $4^{\circ}$ e $5^{\circ}$ mês. Portanto, estes dois pacientes tiveram que ser excluídos da análise de comportamento pela técnica da Análise de Variância com medidas repetidas. Não conseguimos identificar a causa da uveíte.

A evolução da medida da espessura macular central está demonstrado no gráfico 1 . O comportamento do grupo Laser, ao longo das avaliações realizadas, diferiu dos grupos TA $(p=0,0028)$ e Laser+TA $(p=0,0108)$. Entretanto, estes dois grupos não apresentaram diferenças significativas entre si em relação aos seus comportamentos $(\mathrm{p}=0,2639)$ e às suas médias nos diferentes momentos analisados $(\mathrm{p}=0,4839)$. Em relação à medida pré-tratamento, o grupo Laser não apresentou uma redução significativa da espessura foveal ao longo do tempo $(p=0,0826)$. Esta redução foi considerada significativa para os outros dois grupos $(\mathrm{p}<0,001)$.
$\mathrm{O}$ gráfico 2 mostra a evolução do volume macular. $\mathrm{O}$ grupo Laser diferiu dos grupos TA $(\mathrm{p}=0,0122)$ e Laser+TA $(\mathrm{p}=0,0269)$ em relação ao comportamento ao longo das avaliações. Entretanto, estes dois últimos grupos não apresentaram diferenças significativas entre si tanto em relação aos seus comportamentos $(\mathrm{p}=0,2581)$ quanto as suas médias $(p=0,7143)$. Em relação aos valores basais, apenas o grupo Laser não apresentou uma redução significativa do volume macular após tratamento (grupo Laser: $\mathrm{p}=0,11$; grupo TA e grupo Laser+TA: $\mathrm{p}<0,01$ ).

O estudo da acuidade visual pela Análise de Variância com medidas repetidas demonstrou que houve uma melhora significativa da visão ao longo dos meses para os três grupos $(\mathrm{p}=0,0030)$. Não foram encontradas diferenças significativas entre as médias dos diferentes grupos $(\mathrm{p}=0,6047)$. Entretanto, quando foram analisadas as proporções de ganhos e perdas de

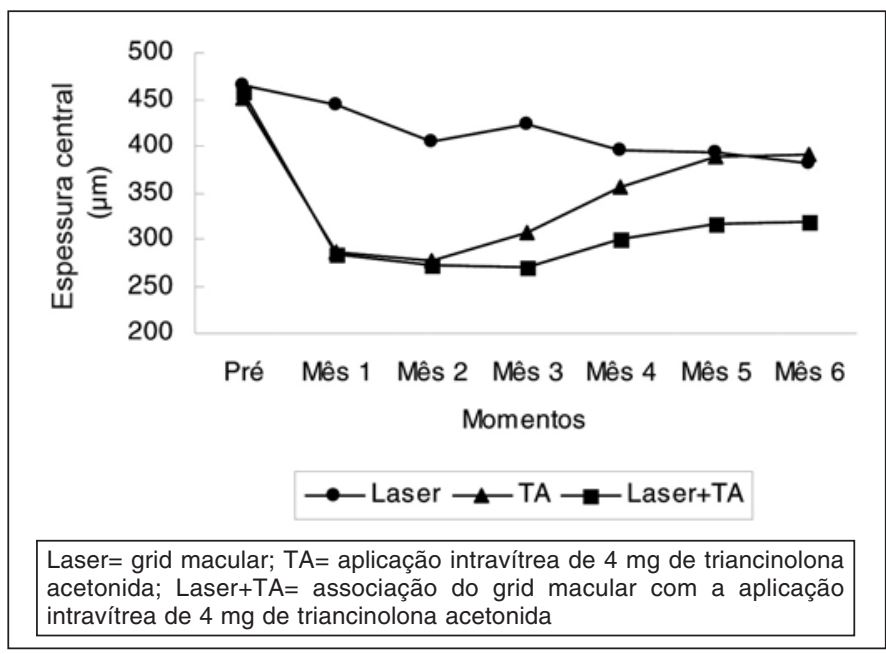

Gráfico 1 - Distribuição das médias da espessura macular central, ao longo dos meses, segundo tipo de tratamento

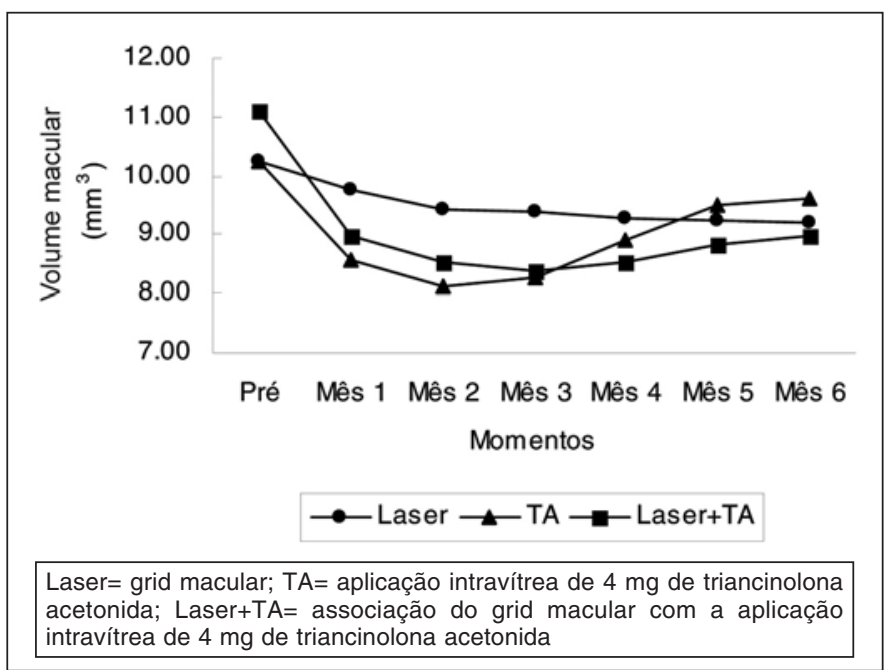

Gráfico 2 - Distribuição das médias do volume macular, ao longo dos meses, segundo tipo de tratamento 


\begin{tabular}{|c|c|c|c|c|c|c|c|c|c|}
\hline \multirow{3}{*}{$\begin{array}{l}\text { Classificação da } \\
\text { acuidade visual }\end{array}$} & \multicolumn{9}{|c|}{ Grupos de tratamento } \\
\hline & \multicolumn{3}{|c|}{ Mês 1} & \multicolumn{3}{|c|}{$\begin{array}{c}\text { Mês 2 } \\
\end{array}$} & \multicolumn{3}{|c|}{ Mês 3} \\
\hline & Laser & TA & Laser+TA & Laser & TA & Laser+TA & Laser & TA & Laser+TA \\
\hline Perda de 10 ou mais letras & 0 & 0 & 0 & 0 & 0 & 0 & 0 & 0 & 0 \\
\hline Perda de 5 a 9 letras & 1 & 1 & 0 & 1 & 1 & 0 & 1 & 1 & \\
\hline Estável & 6 & 7 & 6 & 3 & 3 & 5 & 4 & 5 & 4 \\
\hline Ganho de 5 a 9 letras & 3 & 2 & 2 & 6 & 5 & 4 & 5 & 4 & 2 \\
\hline Ganho de 10 ou mais letras & 0 & 0 & 2 & 0 & 1 & 1 & 0 & 0 & 4 \\
\hline Total & 10 & 10 & 10 & 10 & 10 & 10 & 10 & 10 & 10 \\
\hline
\end{tabular}

\begin{tabular}{|c|c|c|c|c|c|c|c|c|c|}
\hline \multirow{3}{*}{$\begin{array}{l}\text { Classificação da } \\
\text { acuidade visual }\end{array}$} & \multicolumn{9}{|c|}{ Grupos de tratamento } \\
\hline & \multicolumn{3}{|c|}{ Mês 4} & \multicolumn{3}{|c|}{ Mês 5} & \multicolumn{3}{|c|}{ Mês 6} \\
\hline & Laser & TA & Laser+TA & Laser & TA & Laser+TA & Laser & TA & Laser+TA \\
\hline Perda de 10 ou mais letras & 1 & 0 & 0 & 1 & 1 & 0 & 1 & 0 & 0 \\
\hline Perda de 5 a 9 letras & 0 & 3 & 1 & 0 & 0 & 1 & 1 & 3 & 0 \\
\hline Estável & 3 & 3 & 4 & 2 & 6 & 3 & 3 & 4 & 3 \\
\hline Ganho de 5 a 9 letras & 6 & 4 & 1 & 7 & 2 & 2 & 5 & 1 & 5 \\
\hline Ganho de 10 ou mais letras & 0 & 0 & $4^{*}$ & 0 & 0 & $3^{* *}$ & 0 & 1 & 1 \\
\hline Total & 10 & 10 & 10 & 10 & 9 & 9 & 10 & 9 & 9 \\
\hline
\end{tabular}

letras de visão (Tabelas 1 e 2 ), foi observado que no $4^{\circ}$ e $5^{\circ}$ mês de seguimento o grupo Laser+TA apresentou, de forma significativa, maior percentagem de pacientes com ganho de 10 ou mais letras do que os outros dois grupos (Tabela 2).

A pressão intra-ocular elevou-se em $5 \mathrm{mmHg}$ ou mais em 6 pacientes do grupo TA, seis do grupo Laser+TA e nenhum do grupo Laser $(\mathrm{P}<0,001)$. Entre os pacientes que obtiveram uma PIO maior que $21 \mathrm{mmHg}$ após tratamento, 5 estão no grupo TA, 4 no grupo Laser+TA e 1 no grupo Laser $(p=0,228)$. Estes pacientes foram controlados com maleato de timolol $0,5 \%$.

Foi observada progressão da catarata em 4 pessoas do grupo TA, 2 pessoas do grupo Laser e 1 pessoa do grupo Laser+TA. Esta diferença não foi estatisticamente significativa $(p=0,194)$. A progressão da catarata foi considerada clinicamente irrelevante nestes casos.

\section{DISCUSSÃO}

Vários autores relataram que a TA aplicada tanto via intravítrea quanto via subtenoniana pode reduzir o edema macular diabético de forma significativa ${ }^{(10,15-18)}$. Uma série com 38 portadores de EMD, demonstrou que $4 \mathrm{mg}$ de IVTA foi capaz de reduzir a espessura macular basal em $33,8 \%$ no $1^{\circ}$ mês e em $40,1 \%$ no $3^{\circ}$ mês de seguimento ${ }^{(18)}$. Entretanto, tanto reduções maiores $^{(16)}$, de cerca de $60 \%$, como reduções menores ${ }^{(10)}$ de aproximadamente $30 \%$ já foram relatadas. Em nosso estudo, observamos que $4 \mathrm{mg}$ de IVTA reduziu a espessura macular central em $35 \%$ após um mês e $30 \%$ após três meses de tratamento. Assim como registrado por Avitabile et al. ${ }^{(10)}$, a redução que encontramos no grupo submetido a IVTA foi significativamente maior do que a do grupo tratado com laser, que obteve $4,6 \%$ de redução no $1^{\circ}$ mês e $8,9 \%$ no $3^{\circ}$ mês. As reduções da espessura macular central do grupo submetido a associação de laser com IVTA foram um pouco maiores do que a do grupo submetido apenas a IVTA, entretanto, não houve diferença estatisticamente significativa.

Além da regressão parcial do edema macular diabético, a IVTA pode melhorar a acuidade visual destes pacientes ${ }^{(4,10,15,17,19-20)}$. Esta melhora pode ser observada já na primeira semana pós-tratamento ${ }^{(20)}$. Entretanto, assim como acontece na espessura macular, o efeito é transitório, durando cerca de cinco a nove meses ${ }^{(10,16,18,20)}$. Na tentativa de prolongar o benefício do tratamento, alguns autores associaram o grid macular três semanas após a $\operatorname{IVTA}^{(13)}$ e observaram que estes pacientes, quando comparados aos que receberam apenas IVTA, apresentaram maior ganho de visão após três e seis meses de seguimento. Estes mesmos autores relatam que uma das limitações do trabalho foi o uso das tabelas de Snellen para a medida da acuidade visual. Outros autores também afirmaram desconhecer se a realização da IVTA no mesmo dia do laser pudesse proporcionar os mesmos resultados que eles ob- 
servaram no trabalho. Em nosso estudo, fizemos a aplicação do laser e da TA no mesmo dia e utilizamos a medida da acuidade visual preconizada no ETDRS, que é considerada a mais adequada atualmente para este tipo de estudo(2).

A porcentagem de pacientes que melhoram a visão após IVTA e a magnitude desta melhora varia conforme os trabalhos. Num estudo retrospectivo com 19 olhos submetidos a $4 \mathrm{mg}$ de IVTA $^{(15)}$, apenas um olho perdeu uma linha de visão, enquanto que $36,3 \%$ dos olhos ficaram estáveis, $68,4 \%$ melhoraram pelo menos uma linha de visão e $26,3 \%$ melhoraram pelo menos duas linhas. Outros autores, em séries de casos com 12 pacientes, observaram um ganho de duas ou mais linhas de visão em 33 a $42 \%$ dos olhos tratados ${ }^{(16,20)}$. Por outro lado, estudo prospectivo conduzido em três meses, duplo-cego, randomizado e controlado, observaram que 55\% (18/33) dos olhos submetidos a $4 \mathrm{mg}$ de IVTA ganharam cinco ou mais letras de visão, com uma melhora média de 5,1 letras $^{(17)}$. O ganho de 10 ou mais letras foi registrado em $24 \%$ (8/33) dos casos e $42 \%$ (14/33) mantiveramse estáveis. A perda de cinco ou mais letras foi notada em apenas um caso. Entretanto, quando o seguimento destes pacientes foi estendido para dois anos, observou-se que mais quatro olhos perderam cinco ou mais letras de visão, mesmo com re-tratamento permitido a cada 6 meses ${ }^{(19)}$. Em nosso estudo, observamos uma melhora significativa da visão em todos os três grupos de tratamento. O grupo que recebeu a associação de tratamentos apresentou uma maior quantidade de pessoas com ganho de 10 ou mais letras do que os outros dois grupos. É importante ressaltar que nossos pacientes estavam clinicamente compensados do diabetes e da hipertensão arterial. Não sabemos a influência que isto pode ter tido nos resultados de nosso estudo. Talvez pacientes com controle clínico inadequado obtenham piores respostas ao tratamento.

A principal complicação relatada pós IVTA é a elevação da PIO. A freqüência deste aumento varia conforme os trabalhos, oscilando entre $30-50 \%$, e geralmente ocorre entre o $2^{\circ}$ e $3^{\circ}$ mês ${ }^{(17,21-23)}$. Em nosso estudo, $50 \%$ dos pacientes do grupo TA e $40 \%$ dos pacientes do grupo Laser+TA apresentaram uma $\mathrm{PIO}>21 \mathrm{mmHg}$.

A maioria dos trabalhos publicados, até o momento, sobre o uso da IVTA no edema macular diabético, não apresenta grupo controle e não compara a eficácia das opções terapêuticas existentes, apenas analisam os dados de acuidade visual e espessura macular pré e pós-tratamento. Neste trabalho, estudamos e comparamos três tipos de tratamento para o edema macular diabético. Observamos que o grupo de pacientes submetido à associação da IVTA com o grid macular apresentou um maior percentual de ganho de 10 ou mais letras de visão do que os outros grupos. Estudos maiores são necessários para que se possa definir melhor o papel desta associação terapêutica para a população.

\section{ABSTRACT}

Purpose: To assess the efficacy of intravitreal use of triamcinolone acetonide combined with laser photocoagulation for the treatment of diffuse diabetic macular edema and to compare it with the separate use of each treatment. Methods: After systemic clinical control, thirty patients with diffuse diabetic macular edema were divided into 3 treatment groups: (1) macular grid photocoagulation; (2) intravitreal injection of $4 \mathrm{mg}$ of triamcinolone acetonide; (3) combination of the two previous therapies. Follow-up was scheduled at predetermined intervals of one day, one week and monthly until completion of six months. The following parameters were analyzed: LogMAR best corrected visual acuity, central macular thickness, total macular volume and intraocular pressure. Results: Grid photocoagulation did not significantly reduce the central macular thickness or the total macular volume. On the other hand, this reduction was statistically significant in the other two groups. All groups improved their mean visual acuity, however, the group that received both treatments had a higher percentage of patients that gained 10 or more letters. Conclusion: The simultaneous administration of grid photocoagulation with intravitreal triamcinolone can be considered an option for the treatment of diffuse diabetic macular edema.

Keywords: Diabetic retinopathy/complications; Visual acuity; Diabetes complications; Macular edema/drug therapy; Triamcinolone acetonide/therapeutic use; Triamcinolone acetonide/ administration \& dosage; Laser coagulation

\section{REFERÊNCIAS}

1. Resnikoff S, Pascolini D, Etya'ale D, Kocur I, Pararajasegaram R, Pokharel GP, Mariotti SP. Global data on visual impairment in the year 2002. Bull World Health Organ. 2004;82(11):844-51.

2. Photocoagulation for diabetic macular edema. Early Treatment Diabetic Retinopathy Study report number 1. Early Treatment Diabetic Retinopathy Study Research Group. Arch Ophthalmol. 1985;103(12):1796-806.

3. Bresnick GH. Diabetic maculopathy. A critical review highlighting diffuse macular edema. Ophthalmology. 1983;90(11):1301-17.

4. Jonas JB, Kreissig I, Söfker A, Degenring RF. Intravitreal injection of triamcinolone for diffuse diabetic macular edema. Arch Ophthalmol. 2003;121(1):57-61.

5. Han DP, Mieler WF, Burton TC. Submacular fibrosis after photocoagulation for diabetic macular edema. Am J Ophthalmol. 1992;113(5):513-21.

6. Schatz H, Madeira D, McDonald HR, Johnson RN. Progressive enlargement of laser scars following grid laser photocoagulation for diffuse diabetic macular edema. Arch Ophthalmol. 1991;109(11):1549-51.

7. Chalkley TH. The management of ocular inflammation with triamcinolone acetonide. Am J Ophthalmol. 1965;59:815-9.

8. Ip MS, Gottlieb JL, Kahana A, Scott IU, Altaweel MM, Blodi BA, et al. Intravitreal triamcinolone for the treatment of macular edema associated with central retinal vein occlusion. Arch Ophthalmol. 2004;122(8):1131-6.

9. Benhamou N, Massin P, Haouchine B, Audren F, Tadayoni R, Gaudric A. Intravitreal triamcinolone for refractory pseudophakic macular edema. Am J Ophthalmol. 2003;135(2):246-9.

10. Avitabile T, Longo A, Reibaldi A. Intravitreal triamcinolone compared with macular laser grid photocoagulation for the treatment of cystoid macular edema. Am J Ophthalmol. 2005;140(4):695-702.

11. Verma LK, Vivek MB, Kumar A, Tewari HK, Venkatesh P. A prospective controlled trial to evaluate the adjunctive role of posterior subtenon triamcinolone in the treatment of diffuse diabetic macular edema. J Ocul Pharmacol Ther. 2004;20(4):277-84.

12. Tunc M, Onder HI, Kaya M. Posterior sub-Tenon's capsule triamcinolone injection combined with focal laser photocoagulation for diabetic macular edema. 
Ophthalmology. 2005;112(6):1086-91. Comment in: Ophthalmology. 2006; 113(5):885; author reply 885-6.

13. Kang SW, Sa HS, Cho HY, Kim JI. Macular grid photocoagulation after intravitreal triamcinolone acetonide for diffuse diabetic macular edema. Arch Ophthalmol. 2006;124(5):653-8

14. Aiello LP, Brucker AJ, Chang S, Cunningham ET Jr, D’Amico DJ, Flynn HW Jr, et al. Evolving guidelines for intravitreous injections. Retina. 2004; 24(5 Suppl):S3-19. Review.

15. Bakri SJ, Beer PM. Intravitreal triamcinolone injection for diabetic macular edema: a clinical and fluorescein angiographic case series. Can J Ophthalmol. 2004; 39(7):755-60.

16. Massin P, Audren F, Haouchine B, Erginay A, Bergmann JF, Benosman R, et al. Intravitreal triamcinolone acetonide for diabetic diffuse macular edema: preliminary results of a prospective controlled trial. Ophthalmology. 2004;111(2):218-24; discussion 224-5.

17. Sutter FK, Simpson JM, Gillies MC. Intravitreal triamcinolone for diabetic macular edema that persists after laser treatment: three-month efficacy and safety results of a prospective, randomized, double-masked, placebo-controlled clinical trial. Ophthalmology. 2004;111(11):2044-9.

18. Gibran SK, Cullinane A, Jungkim S, Cleary PE. Intravitreal triamcinolone for diffuse diabetic macular oedema. Eye. 2006;20(6):720-4.

19. Gillies MC, Sutter FK, Simpson JM, Larsson J, Ali H, Zhu M. Intravitreal triamcinolone for refractory diabetic macular edema: two-year results of a doublemasked, placebo-controlled, randomized clinical trial. Ophthalmology. 2006; 113(9): $1533-8$

20. Khairallah M, Zeghidi H, Ladjimi A, Yahia SB, Attia S, Zaouali S, Messaoud R. Primary intravitreal triamcinolone acetonide for diabetic massive macular hard exudates. Retina. 2005;25(7):835-9.

21. Jonas JB, Kreissig I, Degenring R. Intraocular pressure after intravitreal injection of triamcinolone acetonide. Br J Ophthalmol. 2003;87(1):24-7.

22. Bakri SJ, Beer PM. The effect of intravitreal triamcinolone acetonide on intraocular pressure. Ophthalmic Surg Lasers Imaging. 2003;34(5):386-90.

23. Smithen LM, Ober MD, Maranan L, Spaide RF. Intravitreal triamcinolone acetonide and intraocular pressure. Am J Ophthalmol. 2004;138(5):740-3. 